Ибавов Т.И. Решение начальной задачи для одной системы трёх дифференциальных уравнений с производной дробного порядка

УДК 517.968

DOI: $10.21779 / 2542-0321-2018-33-1-78-84$

\title{
Т.И. Ибавов
}

\section{Решение начальной задачи для одной системы трёх дифференциальных уравнений с производной дробного порядка}

Дагестанский государственньй университет; Россия, 367001, Махачкала, ул. М. Гаджиева, 43a; ibavov94@mail.ru

В работе исследована задача Коши для системы трех дифференциальных уравнений с производными дробного порядка Caputo. С помощью преобразований Лапласа получены образы функций $x(p), y(p), z(p)$. Для нахождения решения задачи выражения для образов функций $x(t), y(t), z(t)$ разложены на простые дроби с помощью корней характеристического уравнения. Найдено решение Задачи Коши. Получены некоторые соотношения для функции МиттагаЛеффлера, позволяющие упростить полученное решение.

Ключевые слова: дробная производная, дифференциальное уравнение, преобразования Лапласа, функиия Миттага-Леффлера.

\section{Введение}

Учет нелокальных эффектов в рамках традиционных подходов приводит к появлению в дифференциальных уравнениях интегрального оператора, где ядра интегральных операторов несут информацию о природе нелокальности. Для решения подобных уравнений интегральные операторы представляются в виде ряда дифференциальных операторов с возрастающими показателями порядка дифференцирования и при наличии малого параметра ограничиваются несколькими членами ряда. В отсутствии малого параметра такой подход оказывается непродуктивным, и, кроме того, полученные уравнения также не всегда удается решить.

Операция дробного дифференцирования, представляя определенную совокупность операций дифференцирования и интегрирования, открывает новый подход к теории нелокальных дифференциальных уравнений, вносит новый уровень понимания динамики соотношения необратимых и обратимых процессов, когда существенно учтены нелокальные свойства системы. Дробное исчисление вносит в теорию дополнительные параметры в виде показателей дробной производной, дает возможность использования широкого класса многопараметрических функций и открывает тем самым принципиально новые возможности интерпретации экспериментальных данных и создания адекватных количественных моделей процессов нелокального переноса [3].

Решению задач типа Коши для дифференциального уравнения дробного порядка посвящено множество различных работ. В монографии [3] приведены решения задачи Коши для осцилляторных уравнений с производным дробного порядка РиманаЛиувилля и Caputo. В работе [4] исследована начальная задача для фрактального осциллятора, а в работе [5] исследована задача Коши для системы двух линейных дифференциальных уравнений дробного порядка. Работа [6] посвящена исследованию решения задачи Коши для обобщенного уравнения Эйри. Исследованию нелинейных дина- 
Ибавов Т.И. Решение начальной задачи для одной системы трёх дифференциальных уравнений с производной дробного порядка

мических систем, описываемых дифференциальными уравнениями с производными дробного порядка посвящены работы $[7,8,9,10]$.

В данной работе исследована начальная задача для одной системы трех дифференциальных уравнений дробного порядка.

Задача Коши для системы трех дифференциальных уравнений дробного порядка.

Рассмотрим в области $D_{\alpha}$ задачу Коши для системы трех дифференциальных уравнений с производными дробного порядка вида:

$$
\left\{\begin{array}{l}
\partial_{0 t}^{a}=-y-z \\
\partial_{0 t}^{a}=x+a y \\
\partial_{0 t}^{a}=k x+q z,
\end{array}\right.
$$

где $0 \leq \alpha<1, \quad a, q, k-\quad$ некоторые постоянные параметры, $\partial_{0 t}^{\alpha} u(t)=\frac{1}{\Gamma(1-\alpha)} \int_{0(t-s)}^{t} \frac{u^{\prime}(s)}{\alpha} d s-$ дробная производная Сарuto.

Постановка задачи. Найти решение $x(t), y(t), z(t) \in A C^{2}[0, T]$ системы дифференциальных уравнений (1), удовлетворяющее начальным условиям $x(0)=$ $x_{0}, y(0)=y_{0}, z(0)=z_{0}$.

Пусть $D_{\alpha} \subset A C^{2}[0, T]-$ область определения $\partial_{0 t}^{a}$. Тогда для любых функций $x(t) \in D_{\alpha}, y(t) \in D_{\alpha}, z(t) \in D_{\alpha} \quad$ справедливы равенства [2]:

$$
\left\{\begin{array}{l}
D_{0 t}^{a}=-y-z+\frac{x(0)}{\Gamma(1-\alpha) t^{\alpha}}, \\
D_{0 t}^{a}=x+a y+\frac{y(0)}{\Gamma(1-\alpha) t^{\alpha}}, \\
D_{0 t}^{a}=k x+q z+\frac{z(0)}{\Gamma(1-\alpha) t^{\alpha}} .
\end{array}\right.
$$

Пусть $x(t) \in D_{\alpha}, y(t) \in D_{\alpha}, z(t) \in D_{\alpha}$ - решение системы (2). Тогда образы Лапласа системы (2) имеют следующий вид:

$$
\left\{\begin{array}{c}
x(p)=\frac{x_{0} p^{2 \alpha}-p^{\alpha}\left(x_{0} q+x_{0} a+z_{0}+y_{0}\right)+a q x_{0}+z_{0} a+q y_{0}}{p^{1-\alpha}\left[p^{3 \alpha}-p^{2 \alpha}(q+a)+p^{\alpha}(a q+k+1)-(a k+q)\right]} \\
y(p)=\frac{y_{0} p^{2 \alpha}-p^{\alpha}\left(x_{0} q-x_{0}\right)-z_{0}-q x_{0}+k y_{0}}{p^{1-\alpha}\left[p^{3 \alpha}-p^{2 \alpha}(q+a)+p^{\alpha}(a q+k+1)-(a k+q)\right]} \\
z(p)=\frac{z_{0} p^{2 \alpha}-p^{\alpha}\left(z_{0} a-k x_{0}\right)+z_{0}-a k x_{0}-k y_{0}}{p^{1-\alpha}\left[p^{3 \alpha}-p^{2 \alpha}(q+a)+p^{\alpha}(a q+k+1)-(a k+q)\right]}
\end{array}\right.
$$

Представим систему (3) в виде: 
Ибавов Т.И. Решение начальной задачи для одной системы трёх дифференциальных уравнений с производной дробного порядка

$$
\begin{aligned}
& x(p)=\frac{1}{\left(\lambda_{1}-\lambda_{2}\right)\left(\lambda_{1}-\lambda_{3}\right)\left(\lambda_{2}-\lambda_{3}\right)}\left[\frac{x_{0}}{p^{1-2 \alpha}}\left[\frac{1}{1-\lambda_{1} p^{-\alpha}}-\frac{1}{1-\lambda_{2} p^{-\alpha}}+\frac{1}{1-\lambda_{3} p^{-\alpha}}\right]-\right. \\
& -\frac{x_{0} q+x_{0} a+z_{0}+y_{0}}{p^{1-\alpha}}\left[\frac{1}{1-\lambda_{1} p^{-\alpha}}-\frac{1}{1-\lambda_{2} p^{-\alpha}}+\frac{1}{1-\lambda_{3} p^{-\alpha}}\right]+ \\
& +\frac{x_{0} a q+z_{0} a+q y_{0}}{p}\left[\frac{1}{1-\lambda_{1} p^{-\alpha}}-\frac{1}{1-\lambda_{2} p^{-\alpha}}+\frac{1}{1-\lambda_{3} p^{-\alpha}}\right] \text {, } \\
& y(p)=\frac{1}{\left(\lambda_{1}-\lambda_{2}\right)\left(\lambda_{1}-\lambda_{3}\right)\left(\lambda_{2}-\lambda_{3}\right)}\left[\frac{y_{0}}{p^{1-2 \alpha}}\left[\frac{1}{1-\lambda_{1} p^{-\alpha}}-\frac{1}{1-\lambda_{2} p^{-\alpha}}+\frac{1}{1-\lambda_{3} p^{-\alpha}}\right]-\right. \\
& -\frac{y_{0} q-x_{0}}{p^{1-\alpha}}\left[\frac{1}{1-\lambda_{1} p^{-\alpha}}-\frac{1}{1-\lambda_{2} p^{-\alpha}}+\frac{1}{1-\lambda_{3} p^{-\alpha}}\right]+ \\
& \left.+\frac{k y_{0}-x_{0} q-z_{0}}{p}\left[\frac{1}{1-\lambda_{1} p^{-\alpha}}-\frac{1}{1-\lambda_{2} p^{-\alpha}}+\frac{1}{1-\lambda_{3} p^{-\alpha}}\right]\right] \text {, } \\
& z(p)=\frac{1}{\left(\lambda_{1}-\lambda_{2}\right)\left(\lambda_{1}-\lambda_{3}\right)\left(\lambda_{2}-\lambda_{3}\right)}\left[\frac{z_{0}}{p^{1-2 \alpha}}\left[\frac{1}{1-\lambda_{1} p^{-\alpha}}-\frac{1}{1-\lambda_{2} p^{-\alpha}}+\frac{1}{1-\lambda_{3} p^{-\alpha}}\right]-\right. \\
& -\frac{z_{0} a-k x_{0}}{p^{1-\alpha}}\left[\frac{1}{1-\lambda_{1} p^{-\alpha}}-\frac{1}{1-\lambda_{2} p^{-\alpha}}+\frac{1}{1-\lambda_{3} p^{-\alpha}}\right]+ \\
& +\frac{z x_{0}-a x_{0} k-k y_{0}}{p}\left[\frac{1}{1-\lambda_{1} p^{-\alpha}}-\frac{1}{1-\lambda_{2} p^{-\alpha}}+\frac{1}{1-\lambda_{3} p^{-\alpha}}\right] \text {, }
\end{aligned}
$$

где, $\lambda_{1}, \lambda_{2}, \lambda_{3}-$ корни характеристического уравнения.,

$$
\begin{gathered}
\lambda_{1}=\sqrt[3]{-\beta+\sqrt{\beta^{2}+\gamma^{3}}}+\sqrt[3]{-\beta-\sqrt{\beta^{2}+\gamma^{3}}}-\frac{q+a}{3}, \\
\lambda_{2}=\varepsilon_{1} \sqrt[3]{-\beta+\sqrt{\beta^{2}+\gamma^{3}}}+\varepsilon_{2} \sqrt[3]{-\beta-\sqrt{\beta^{2}+\gamma^{3}}}-\frac{q+a}{3}, \\
\lambda_{3}=\varepsilon_{2} \sqrt[3]{-\beta+\sqrt{\beta^{2}+\gamma^{3}}}+\varepsilon_{1} \sqrt[3]{-\beta-\sqrt{\beta^{2}+\gamma^{3}}}-\frac{q+a}{3}, \\
\text { где } \beta=\frac{\frac{q+a}{3}[a q+k+1]-k a-q-\frac{2}{27}[q+a]^{3}}{2}, \gamma=\frac{a q+k+1-\frac{1}{3}[q+a]^{2}}{27}, \\
\mathcal{E}_{1}, \boldsymbol{E}_{2}=-\frac{1}{2} \pm \frac{\sqrt{3}}{2} .
\end{gathered}
$$


Приведём некоторые вспомогательные соотношения из [3]:

$$
\begin{gathered}
\int_{0}^{\infty} e^{-t_{t} \beta-1} E_{\alpha, \beta}\left(t^{\alpha} z\right) d t=\frac{1}{1-z}, \quad|z|<1, \\
\frac{1}{p\left(1-\lambda \cdot p^{-\alpha}\right)}=\int_{0}^{\infty} e^{-p t} E_{\alpha, 1}\left(\lambda t^{\alpha}\right) d t, \quad \operatorname{Re} p>1, \\
\frac{1}{p^{1-\alpha}\left(1-\lambda \cdot p^{-\alpha}\right)}=\int_{0}^{\infty} e^{-p t} t^{-\alpha} E_{\alpha, 1-\alpha}\left(\lambda t^{\alpha}\right) d t, \operatorname{Re} p>1 .
\end{gathered}
$$

из равенств $(4 a),(4 \sigma),(4 c)$ с помощью соотношений (5) получим выражения для оригиналов функций $x(t), y(t), z(t)$ :

$$
\begin{aligned}
& x(t)=\frac{1}{\left(\lambda_{1}-\lambda_{2}\right)\left(\lambda_{1}-\lambda_{3}\right)\left(\lambda_{2}-\lambda_{3}\right)}\left(x _ { 0 } \mathrm { t } ^ { - 2 \alpha } \left(\left(\lambda_{2}-\lambda_{3}\right) \mathrm{E}_{\alpha, 1-2 \alpha}\left(\lambda_{1} t^{\alpha}\right)-\right.\right. \\
& \left.-\left(\lambda_{2}-\lambda_{3}\right) \mathrm{E}_{\alpha, 1-2 \alpha}\left(\lambda_{1} t^{\alpha}\right)+\left(\lambda_{1}-\lambda_{2}\right) \mathrm{E}_{\alpha, 1-2 \alpha}\left(\lambda_{1} t^{\alpha}\right)\right)-\left(x_{0} q+x_{0} a+z_{0}+y_{0}\right) \\
& \left.t^{-\alpha}\left(\left(\lambda_{2}-\lambda_{3}\right) \mathrm{E}_{\alpha, 1-\alpha}\left(\lambda_{1} t^{\alpha}\right)-\left(\lambda_{2}-\lambda_{3}\right) \mathrm{E}_{\alpha, 1-\alpha}\left(\lambda_{1} t^{\alpha}\right)+\left(\lambda_{1}-\lambda_{2}\right) \mathrm{E}_{\alpha, 1-\alpha}\left(\lambda_{1} t^{\alpha}\right)\right)\right)+ \\
& +\left(x_{0} a q+z_{0} a+q y_{0}\right)\left(\left(\lambda_{2}-\lambda_{3}\right) \mathrm{E}_{\alpha, 1}\left(\lambda_{1} t^{\alpha}\right)-\left(\lambda_{2}-\lambda_{3}\right) \mathrm{E}_{\alpha, 1}\left(\lambda_{1} t^{\alpha}\right)+\right. \\
& \left.\left.+\left(\lambda_{1}-\lambda_{2}\right) \mathrm{E}_{\alpha, 1}\left(\lambda_{1} t^{\alpha}\right)\right)\right) \\
& y(t)=\frac{1}{\left(\lambda_{1}-\lambda_{2}\right)\left(\lambda_{1}-\lambda_{3}\right)\left(\lambda_{2}-\lambda_{3}\right)}\left(y _ { 0 } \mathrm { t } ^ { - 2 \alpha } \left(\left(\lambda_{2}-\lambda_{3}\right) \mathrm{E}_{\alpha, 1-2 \alpha}\left(\lambda_{1} t^{\alpha}\right)-\right.\right. \\
& \left.-\left(\lambda_{2}-\lambda_{3}\right) \mathrm{E}_{\alpha, 1-2 \alpha}\left(\lambda_{1} t^{\alpha}\right)+\left(\lambda_{1}-\lambda_{2}\right) \mathrm{E}_{\alpha, 1-2 \alpha}\left(\lambda_{1} t^{\alpha}\right)\right)-\left(y_{0} q-x_{0}\right) \\
& \left.t^{-\alpha}\left(\left(\lambda_{2}-\lambda_{3}\right) \mathrm{E}_{\alpha, 1-\alpha}\left(\lambda_{1} t^{\alpha}\right)-\left(\lambda_{2}-\lambda_{3}\right) \mathrm{E}_{\alpha, 1-\alpha}\left(\lambda_{1} t^{\alpha}\right)+\left(\lambda_{1}-\lambda_{2}\right) \mathrm{E}_{\alpha, 1-\alpha}\left(\lambda_{1} t^{\alpha}\right)\right)\right) \\
& \left(k y_{0}-z_{0}-q x_{0}\right)\left(\left(\lambda_{2}-\lambda_{3}\right) \mathrm{E}_{\alpha, 1}\left(\lambda_{1} t^{\alpha}\right)-\left(\lambda_{2}-\lambda_{3}\right) \mathrm{E}_{\alpha, 1}\left(\lambda_{1} t^{\alpha}\right)+\right. \\
& \left.\left.+\left(\lambda_{1}-\lambda_{2}\right) \mathrm{E}_{\alpha, 1}\left(\lambda_{1} t^{\alpha}\right)\right)\right) \\
& z(t)=\frac{1}{\left(\lambda_{1}-\lambda_{2}\right)\left(\lambda_{1}-\lambda_{3}\right)\left(\lambda_{2}-\lambda_{3}\right)}\left(z _ { 0 } \mathrm { t } ^ { - 2 \alpha } \left(\left(\lambda_{2}-\lambda_{3}\right) \mathrm{E}_{\alpha, 1-2 \alpha}\left(\lambda_{1} t^{\alpha}\right)-\right.\right. \\
& \left.-\left(\lambda_{2}-\lambda_{3}\right) \mathrm{E}_{\alpha, 1-2 \alpha}\left(\lambda_{1} t^{\alpha}\right)+\left(\lambda_{1}-\lambda_{2}\right) \mathrm{E}_{\alpha, 1-2 \alpha}\left(\lambda_{1} t^{\alpha}\right)\right)-\left(z_{0} a-k_{x_{0}}\right) \\
& \left.t^{-\alpha}\left(\left(\lambda_{2}-\lambda_{3}\right) \mathrm{E}_{\alpha, 1-\alpha}\left(\lambda_{1} t^{\alpha}\right)-\left(\lambda_{2}-\lambda_{3}\right) \mathrm{E}_{\alpha, 1-\alpha}\left(\lambda_{1} t^{\alpha}\right)+\left(\lambda_{1}-\lambda_{2}\right) \mathrm{E}_{\alpha, 1-\alpha}\left(\lambda_{1} t^{\alpha}\right)\right)\right) \\
& \left(z_{0}-a k x_{0}-k y_{0}\right)\left(\left(\lambda_{2}-\lambda_{3}\right) \mathrm{E} \alpha, 1\left(\lambda_{1} t^{\alpha}\right)-\left(\lambda_{2}-\lambda_{3}\right) \mathrm{E}_{\alpha, 1}\left(\lambda_{1} t^{\alpha}\right)+\right. \\
& \left.\left.+\left(\lambda_{1}-\lambda_{2}\right) \mathrm{E}_{\alpha, 1}\left(\lambda_{1} t^{\alpha}\right)\right)\right)
\end{aligned}
$$

Лемма 1. Для $\alpha>0$ имеет место равенство

$$
\left.t^{-\alpha} E_{\alpha, 1-\alpha}{ }^{\alpha}{ }^{\alpha}\right)=\frac{1}{\Gamma(1-\alpha) t}{ }^{\alpha}+E_{\alpha, 1}\left({ }^{\alpha}\right) .
$$


Ибавов Т.И. Решение начальной задачи для одной системы трёх дифференциальных уравнений с производной дробного порядка

Доказательство. Согласно определению функции Миттаг-Леффлера имеем

$$
t^{-\alpha} E_{\alpha, 1-\alpha}\left(t^{\alpha}\right)=t^{-\alpha} \sum_{n=0}^{\infty} \frac{t^{\alpha n}}{\Gamma(\alpha n+1-\alpha)}, \alpha>0 .
$$

В правой части равенства (7), заменяя $n$ на $n^{\prime}+1$, получим

$$
\begin{aligned}
& t^{-\alpha} \sum_{n^{\prime}=-1}^{\infty} \frac{t^{\alpha n^{\prime}+\alpha}}{\Gamma\left(\alpha n^{\prime}+\alpha+1-\alpha\right)}=\sum_{n^{\prime}=-1}^{\infty} \frac{t^{\alpha n^{\prime}}}{\Gamma\left(\alpha n^{\prime}+1\right)}= \\
& =\frac{t^{-\alpha}}{\Gamma(1-\alpha)}+\sum_{n^{\prime}=0}^{\infty} \frac{t^{\alpha n^{\prime}}}{\Gamma\left(\alpha n^{\prime}+1\right)}=\frac{t^{-\alpha}}{\Gamma(1-\alpha)}+E_{\alpha, 1^{(}\left(t^{\alpha}\right) .}
\end{aligned}
$$

Лемма 2. Для $\alpha>0$ имеет место равенство

$$
t^{-2 \alpha} E_{\alpha, 1-2 \alpha}\left(t^{\alpha}\right)=\frac{1}{\Gamma(1-2 \alpha) t^{2 \alpha}}+\frac{1}{\Gamma(1-\alpha) t^{\alpha}}+E_{\alpha, 1}\left(t^{\alpha}\right) .
$$

Доказательство. Согласно определению функции Миттаг-Леффлера имеем

$$
\left.t^{-2 \alpha} E_{\alpha, 1-2 \alpha}{ }^{\alpha}{ }^{\alpha}\right)=t^{-2 \alpha} \sum_{n=0}^{\infty} \frac{t^{\alpha n}}{\Gamma(\alpha n+1-2 \alpha)}, \alpha>0 .
$$

В правой части равенства (9), заменяя $n$ на $n^{\prime}+2$, получим

$$
\begin{aligned}
& t^{-2 \alpha} \sum_{n^{\prime}=-1}^{\infty} \frac{t^{\alpha n^{\prime}+2 \alpha}}{\Gamma\left(\alpha n^{\prime}+2 \alpha+1-2 \alpha\right)}=\sum_{n^{\prime}=-1}^{\infty} \frac{t^{\alpha n^{\prime}}}{\Gamma\left(\alpha n^{\prime}+1\right)}= \\
& =\frac{t^{-2 \alpha}}{\Gamma(1-2 \alpha)}+\frac{t^{-\alpha}}{\Gamma(1-\alpha)}+\sum_{n^{\prime}=0}^{\infty} \frac{t^{\alpha n^{\prime}}}{\Gamma\left(\alpha n^{\prime}+1\right)}=\frac{t^{-2 \alpha}}{\Gamma(1-2 \alpha)}+\frac{t^{-\alpha}}{\Gamma(1-\alpha)}+E_{\left.\alpha, 1^{(} t^{\alpha}\right) .}
\end{aligned}
$$

Используя равенства (6) и (8), решение можно представить в виде:

$$
\begin{gathered}
x(t)=\frac{1}{\left(\lambda_{1}-\lambda_{2}\right)\left(\lambda_{1}-\lambda_{3}\right)\left(\lambda_{2}-\lambda_{3}\right)}\left(\left(\lambda_{2}-\lambda_{3}\right) \mathrm{E}_{\alpha, 1}\left(\lambda_{1} t^{\alpha}\right)-\left(\lambda_{1}-\lambda_{3}\right) \mathrm{E}_{\alpha, 1}\left(\lambda_{2} t^{\alpha}\right)+\right. \\
\left.+\left(\lambda_{1}-\lambda_{2}\right) \mathrm{E}_{\alpha, 1}\left(\lambda_{3} t^{\alpha}\right)\right) \cdot\left(x_{0}(1-q-a+a q)+y_{0}(q-1)+z_{0}(a-1)\right), \\
y(t)=\frac{1}{\left(\lambda_{1}-\lambda_{2}\right)\left(\lambda_{1}-\lambda_{3}\right)\left(\lambda_{2}-\lambda_{3}\right)}\left(\left(\lambda_{2}-\lambda_{3}\right) \mathrm{E}_{\alpha, 1}\left(\lambda_{1} t^{\alpha}\right)-\left(\lambda_{1}-\lambda_{3}\right) \mathrm{E}_{\alpha, 1}\left(\lambda_{2} t^{\alpha}\right)+\right. \\
\left.+\left(\lambda_{1}-\lambda_{2}\right) \mathrm{E}_{\alpha, 1}\left(\lambda_{3} t^{\alpha}\right)\right) \cdot\left(y_{0}(1-q+k)-x_{0}(q-1)-z_{0}\right), \quad(10 b) \\
z(t)=\frac{1}{\left(\lambda_{1}-\lambda_{2}\right)\left(\lambda_{1}-\lambda_{3}\right)\left(\lambda_{2}-\lambda_{3}\right)}\left(\left(\lambda_{2}-\lambda_{3}\right) \mathrm{E}_{\alpha, 1}\left(\lambda_{1} t^{\alpha}\right)-\left(\lambda_{1}-\lambda_{3}\right) \mathrm{E}_{\alpha, 1}\left(\lambda_{2} t^{\alpha}\right)+\right. \\
\left.+\left(\lambda_{1}-\lambda_{2}\right) \mathrm{E}_{\alpha, 1}\left(\lambda_{3} t^{\alpha}\right)\right) \cdot\left(z_{0}(2-a)+x_{0} k(1-a)-k y_{0}\right) .
\end{gathered}
$$


Ибавов Т.И. Решение начальной задачи для одной системы трёх дифференциальных уравнений с производной дробного порядка

Имеет место следующая теорема.

Теорема. Функции $x(t) \in D_{\alpha}, y(t) \in D_{\alpha}, z(t) \in D_{\alpha}$ будут решениями системы (1), если они представимы в виде $(9 a),(9 b),(9 c)$.

\section{Литература}

1. Самко С.Г., Килбас А.А., Маричев О.И. Интегралы и производные дробного порядка и некоторые их приложения. - Минск: Наука и техника, 1987. - 498 с.

2. Нахушев А.М. Дробное исчисление и его применение. - М.: Физматлит, 2003. $-272 \mathrm{c}$.

3. Мейланов Р.П., Бейбалаев В.Д., Шахбанова М.Р. Прикладные аспекты дробного исчисления // Palmarium Academic Publishing, Saarbrücken: 2012. - 135 c.

4. Бейбалаев В.Д. Решение начальной задачи для дифференциального уравнения «фрактального» осциллятора // Вестник Самарского гос. тех. ун-та. Сер. Физ.-мат. науки. - 2009. - Т. 1 (19).

5. Назаралиев М.А., Бейбалаев В.Д. Динамические системы, описываемые двумя дифференциальными уравнениями с производными дробного порядка // Владикавказский математический журнал. - 2013. - Т. 15, № 1. - С. 30-40.

6. Паровик Р.И. Задача Коши для обобщенного уравнения Эйри // Доклады AMAН. - 2014. - Т. 16, № 3. - С. 64-69.

7. Бейбалаев В.Д., Мейланов Р.П., Назаралиев М.А., Шабанова М.Р. Особенности фазовой траектории «фрактального» брюсселятора // Сб. материалов седьмой Всероссийской конференции «Математическое моделирование и краевые задачи». - Самара 3-6 июня, 2010. - Самара, 2010.

8. Назаралиев М.А., Бейбалаев В.Д. Нелинейные колебания в средах с фрактальной структурой // Сб. трудов Международного Российско-Болгарского симпозиума, КБР, г. Нальчик, КЧР, а. Хабез 25-30 июня, 2010. - Нальчик, 2010.

9. Бейбалаев В.Д. Моделирование хаотического поведения динамических систем с фрактальной структурой // Сб. материалов восьмой Всероссийской конференции с международным участием «Математическое моделирование и краевые задачи», СамГТУ Самара, 2011. - Самара, 2011. - С. 27-31.

10. Тренькин А.А., Карелин В.И., Алисултанов 3.3., Бейбалаев В.Д., Рагимханов Г.Б. Динамика электронов в ветвящихся системах газоразрядных каналов со спадающей концентрацией газа // Нелинейный мир. - 2017. - Т. 15, № 3. - С. 24-31.

Поступила в редакциюю 27 февраля 2018 г. 
Ибавов Т.И. Решение начальной задачи для одной системы трёх дифференциальных уравнений с производной дробного порядка

UDC 517.968

DOI: $10.21779 / 2542-0321-2018-33-1-78-84$

\title{
The solution of Cauchy problem for system of three differential equations with fractional derivative Caputo
}

\begin{abstract}
T.I. Ibavov
Dagestan State University; Russia, Makhachkala, 367001, M. Gadzhiev st., 43 a; ibavov94@mail.ru

In the paper the Cauchy problem for system of three differential equations with fractional derivative Caputo is studied. The images of decision $x(p), y(p), z(p)$ are obtained using Laplace transforms. To find the solution of the problem $x(t), y(t), z(t)$, the image of solution was decomposed into simple fractions using the roots of characteristic equation. Some relations are obtained for the Mittag-Leffler function, which allow simplifying the obtained solution. The solution of Cauchy problem for system of three differential equation with fractional derivative Caputo is found.
\end{abstract} tion.

Keywords: fractional derivative, differential equation, Laplace transform, Mittag-Leffler func-

Received 27 February, 2018 